\title{
RELACIÓN ENTRE GASTO Y CALIDAD EN LAS UNIVERSIDADES
}

\author{
Jesús M. de Miguel
}

Universidad de Barcelona

\begin{abstract}
RESUMEN
En España, hasta ahora no se podía relacionar la calidad de las universidades con el gasto que suponen. En el año 2002, por fin, se pueden calcular esas dos variables gracias a la publicación de los datos del Instituto Nacional de Estadística y de la CRUE (Conferencia de Rectores de Universidades Españolas). La estrecha relación entre calidad y gasto que demuestran los datos que se incluyen aquí obliga a replantearse la relación entre recursos y resultados, e incluso la supuesta autonomía universitaria. Teniendo en cuenta que son variables diferentes, y calculadas de forma independiente, la relación es más llamativa aún. Sin embargo, hay universidades que se escapan - relativamente- a esa relación causal, lo que permite valorar la importancia de las variables de contexto y de funcionamiento. En la Sociología de las Organizaciones faltan estudios serios sobre la universidad en España. Con la nueva Ley Orgánica de Universidades (6/2001) y el Decreto de Habilitación/Acceso (744/2002), el sistema universitario español (92\% público y $8 \%$ privado) está transformándose más de lo que los sociólogos/as suponen. Los datos de esta nota de investigación resumen un centenar de variables analizadas en el estudio Excelencia: Calidad de las universidades españolas (publicado por el CIS en 2001) y miles de datos concretos de universidades públicas y privadas. Por primera vez, las universidades españolas son evaluadas en su productividad y calidad, observándose una diversidad que supone una creciente competición por los/as mejores estudiantes, profesores, investigadores y PAS. Los datos permiten también avanzar en un debate importante sobre la posibilidad de crear en España un modelo de universidad-investigadora que sea competitiva a nivel internacional. Desgraciadamente, ese cambio sucede en pleno proceso de descentralización universitaria, por lo que el diseño de esas (pocas) universidades-investigadoras de prestigio internacional recaería en las diecisiete Comunidades Autónomas.
\end{abstract}


El análisis de la calidad de la universidad es un tema importante de debate y análisis científico ${ }^{1}$. Justo antes del verano de 2002, la revista Gaceta Universitaria publicó un análisis de la calidad global de las universidades públicas españolas y de los sistemas universitarios de las Comunidades Autónomas ${ }^{2}$. Cientos de personas e instituciones solicitaron información a través del correo electrónico de la Universidad de Barcelona: demiguel@eco.ub.es. Algunas personas querían conocer las relaciones entre los recursos y los resultados de las universidades, y más concretamente entre el gasto y los índices de calidad. En el presente artículo se realiza el análisis de esa relación utilizando los datos recientes publicados, por primera vez en la historia de la universidad española, por las universidades públicas ${ }^{3}$.

Todavía no existen datos públicos oficiales sobre el gasto de las universidades públicas y privadas en España. La CRUE (Conferencia de Rectores de Universidades Españolas) publica en la red un informe, realizado por Juan Hernández Armenteros, con datos financieros de las universidades públicas españolas. El estudio se titula Información académica, productiva y financiera de las universidades públicas españolas. Año 2000: Indicadores universitarios (curso académico 20002001), publicado en $2002^{4}$. Por primera vez es posible comparar los datos de calidad global de las universidades con el gasto efectivo realizado por las universidades. Existe una relación estrecha entre calidad y gasto. No es una sorpresa, pero lo es que las correlaciones sean tan altas. Teniendo en cuenta que los indicadores no se solapan, indica que la medición de la calidad según los indicadores propuestos en el estudio Excelencia, y posteriormente en Calidad de las universidades españolas y sistema universitario de las Comunidades Autónomas, es apropiada 5 .

La relación es estrecha entre la tasa de profesorado y el gasto. La partida presupuestaria más importante es personal, sobre todo personal docente. En el gráfico 1 se puede ver la relación de estas dos variables — tasa de profesorado por cien estudiantes y gasto por estudiante- para las universidades públicas (las privadas no presentan estadísticas de gasto). La correlación es muy alta (coeficiente de correlación de 0,80 ). La tendencia isomórfica de las universidades públicas es considerable. La Universidad Nacional de Educación a Distancia - UNED_, que no es una universidad comparable a las demás por su carácter de «a distancia», aparece retrasada en el gráfico. Las demás univer-

${ }^{1}$ Jesús M. de Miguel, Jordi Caïs y Elizabeth Vaquera, Excelencia: Calidad de las universidades españolas (Madrid: Centro de Investigaciones Sociológicas, Presidencia del Gobierno, 2001), 506 pp., y Calidad de las universidades españolas y sistema universitario de las Comunidades Autónomas (Barcelona: Universidad de Barcelona, septiembre 2002), 102 pp. Véase el análisis crítico de Víctor Pérez Díaz y Juan C. Rodríguez, Educación superior y futuro de España (Madrid: Fundación Santillana, 2001), 470 pp.

2 «Ranking de universidades», Gaceta Universitaria, 413 (24 junio 2002), pp. 1-15.

${ }^{3}$ Los cálculos para el presente artículo han sido realizados con la colaboración de Jordi Caïs (Universidad de Barcelona) y Elizabeth Vaquera (University of Pennsylvania). Una actualización puede obtenerse en demiguel@eco.ub.es.

${ }^{4}$ En la red, en www.crue.org/cdOBSERVATORIO/index.htm (consultado el 3 julio 2002; véase especialmente la tabla V.I.9).

5 Jesús M. de Miguel, Jordi Caïs y Elizabeth Vaquera (2001 y 2002). 


\section{GRÁFICO 1}

Tasa de profesorado y gasto de las universidades públicas

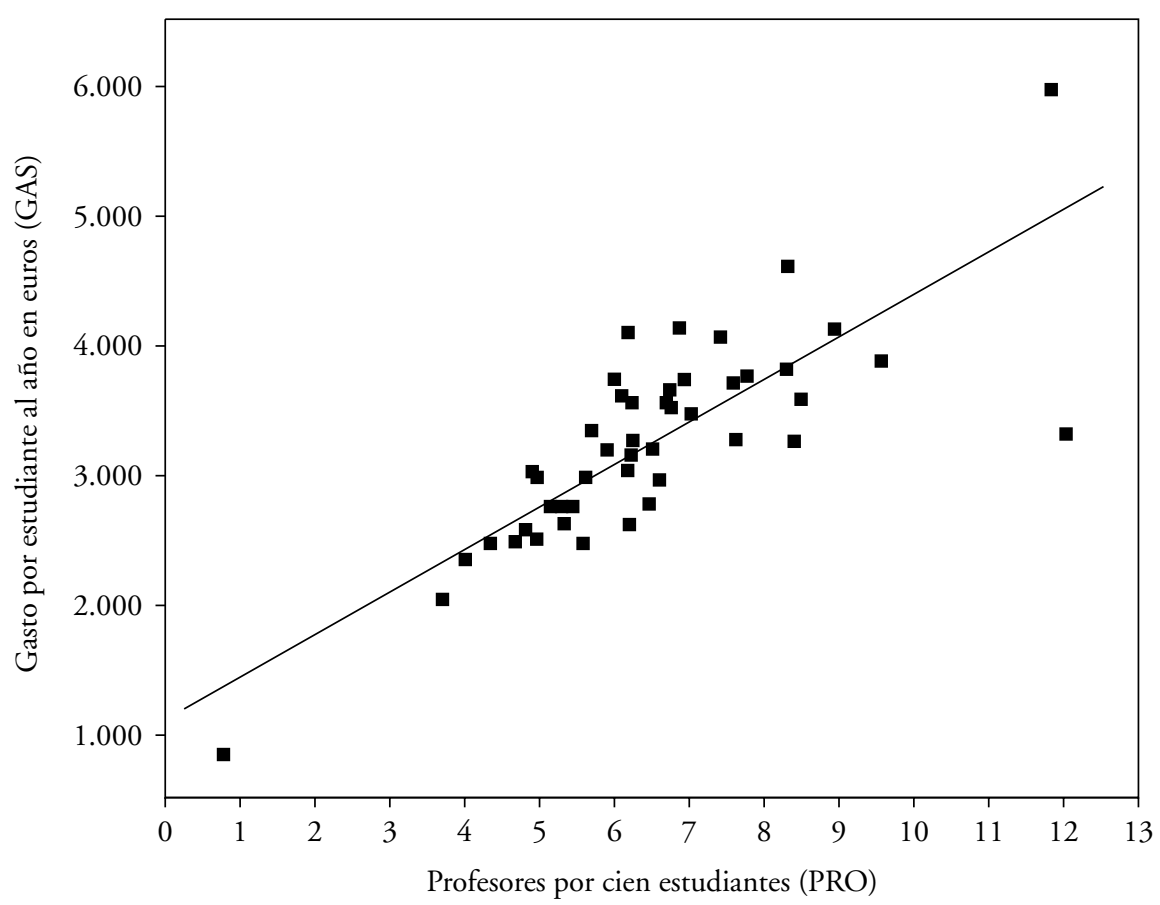

NotA: La correlación entre las variables PRO y GAS es 0,80 .

sidades se agrupan en un núcleo del que sólo se escapa por delante la Universidad Pompeu Fabra, con mucho profesorado y, sobre todo, con un gasto por estudiante que es el doble de la media española. También destaca la Universidad Rey Juan Carlos (en Madrid), con un profesorado comparable a la Pompeu Fabra pero con un gasto per cápita (estudiante) que es la mitad. Las universidades que gastan más de lo esperable según la tasa de profesorado son las politécnicas (Cataluña y Madrid sobre todo), la Complutense, Pública de Navarra y Las Palmas (que es bastante politécnica). El sobregasto se entiende por la biografía característica de esas universidades. En cambio, las universidades que gastan menos de lo previsto según su tasa de profesorado son

${ }^{6}$ La explicación puede verse en «A young university in the Old World: A new Spanish institution reveals the potential for European academic reform" The Chronicle of Higher Education, vol. 49, n. ${ }^{\circ} 11$ (8 noviembre 2002), pp. A40-A42. El gasto por estudiante al año en la Universidad Pompeu Fabra es 5.973 euros, cuando la media española es 3.218 euros. 
Miguel Hernández (en Alicante), que tiene mucho profesorado pero no tanto gasto; Alcalá de Henares, Almería, Coruña y la nueva sevillana de Pablo de Olavide. Pero salvo tres universidades (UNED por debajo, Pompeu Fabra y Rey Juan Carlos por encima), las demás universidades públicas se agrupan en una pauta esperable de gasto y profesorado estrechamente relacionados. La tasa de profesorado se decanta como el indicador más importante del centenar utilizado en los estudios para medir la calidad global de las universidades españolas. Dada esta asociación, es esperable, pues, que calidad y gasto estén muy relacionados.

La relación numérica entre gasto y calidad aparece en la tabla 1, en donde se compara el gasto por estudiante al año (en euros) y la nota global en el ranking de calidad de las universidades públicas. El coste medio es 3.218 euros por estudiante, teniendo en cuenta las universidades públicas. Un punto en el ranking vale 488 euros al año (por estudiante). A simple vista, se observa que las universidades situadas en los diez primeros puestos gastan todas más de 3.000 euros por estudiante al año. La Universidad Pompeu Fabra llega al máximo de casi 6.000 euros, lo que explica la calidad obtenida por esta universidad en apenas unos años. En las universidades de en medio el gasto va bajando

\section{TABLA 1}

Gasto por estudiante en las universidades públicas

\begin{tabular}{|c|c|c|c|}
\hline Universidad & $\begin{array}{l}\text { Gasto por estudiante } \\
\text { al año (en euros) }\end{array}$ & Nota en el ranking & $\begin{array}{l}\text { Coste de un punto } \\
\text { en la nota del ranking }\end{array}$ \\
\hline Complutense de Madrid .............. & 4.073 & 9,6 & 424 \\
\hline Autónoma de Madrid ..................... & 3.692 & 9,5 & 389 \\
\hline 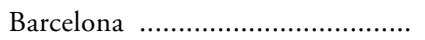 & 3.717 & 9,2 & 404 \\
\hline 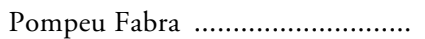 & 5.973 & 9,0 & 663 \\
\hline Autónoma de Barcelona ................ & 4.104 & 8,9 & 461 \\
\hline 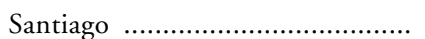 & 3.007 & 8,4 & 358 \\
\hline Alcalá de Henares ............................ & 3.798 & 7,9 & 481 \\
\hline 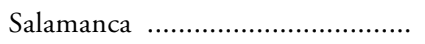 & 3.546 & 7,8 & 455 \\
\hline 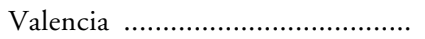 & 3.249 & 7,8 & 416 \\
\hline Rey Juan Carlos ................................ & 3.309 & 7,8 & 424 \\
\hline 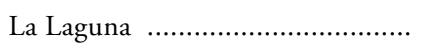 & 3.569 & 7,8 & 458 \\
\hline 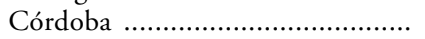 & 3.596 & 7,6 & 473 \\
\hline Miguel Hernández de Elche ......... & 3.861 & 7,4 & 521 \\
\hline 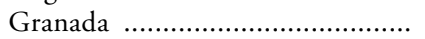 & 2.743 & 7,2 & 381 \\
\hline 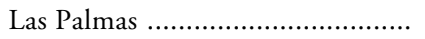 & 3.643 & 6,9 & 528 \\
\hline 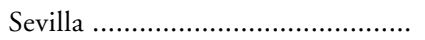 & 2.607 & 6,9 & 378 \\
\hline 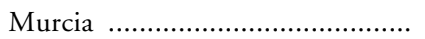 & 2.740 & 6,8 & 403 \\
\hline 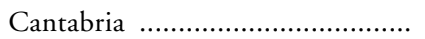 & 3.746 & 6,8 & 551 \\
\hline 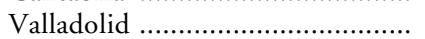 & 3.190 & 6,8 & 469 \\
\hline
\end{tabular}


TABLA 1 (continuación)

Gasto por estudiante en las universidades públicas

\begin{tabular}{lccc}
\hline & $\begin{array}{c}\text { Gasto por estudiante } \\
\text { al año (en euros) }\end{array}$ & & $\begin{array}{c}\text { Coste de un punto } \\
\text { Nota en el ranking }\end{array}$ \\
en la notad del ranking
\end{tabular}

FUENTES: Juan Hernández Armenteros, Información académica, productiva y financiera de las universidades públicas españolas. Año 2000: Indicadores universitarios (curso académico 20002001) (Madrid: Conferencia de Rectores de Universidades Españolas, 2002), http://www.crue. org/cdOBSERVATORIO/index.htm. Consultado el 3 de julio de 2002. Tabla V.I.9. Jesús M. de Miguel, Jordi Caïs y Elisabeth Vaquera, Calidad de las universidades españolas y sistema universitario de las Comunidades Autónomas (Barcelona: Universidad de Barcelona, septiembre 2002). 
desde más de 3.000 hasta los 2.000, con alguna excepción como el gasto de 4.600 euros al año por estudiante en la Universidad Politécnica de Cataluña. En el bloque de las diez universidades situadas más abajo, el coste — salvo en La Rioja - no llega a 3.000 euros al año. En el caso de la UNED es, lógicamente, más bajo aún (con 820 euros al año por estudiante), pero depende de su característica de universidad no presencial. El coeficiente de correlación entre gasto y calidad es 0,68 , que es muy alto para dos variables que no están relacionadas directamente. Los datos de la CRUE permiten por vez primera comprobar que el éxito de una organización compleja (como es la universidad) depende mucho de su presupuesto. El éxito de universidades emblemáticas como Pompeu Fabra, en Barcelona, o Rey Juan Carlos, en Madrid, depende de los recursos económicos adjudicados, y de otras variables básicas como dotación de profesorado. Así se explica también la calidad en otras universidades nuevas como Miguel Hernández de Elche. En las diez universidades más altas en el ranking el coste por punto del ranking (448) es menor que en las diez universidades de menos calidad (524 euros por punto). La variación es casi del doble, desde 358 euros por punto de la Universidad de Santiago (la sexta universidad de España en calidad) hasta 705 euros por punto de la Universidad de La Rioja. Además de ésta, las universidades españolas más caras en la relación coste/calidad son Burgos, Politécnica de Cataluña, Pompeu Fabra, Pública de Navarra y Politécnica de Madrid; todas con un coste de más de 600 euros por punto del ranking.

En el gráfico 2 se puede ver la expresión visual de esa relación. UNED aparece en retaguardia, pero está equilibrada en cuanto a gasto y calidad. Hay que notar que a medida que se aumenta el gasto hay una cierta dispersión de la calidad. Eso significa que el éxito no se debe únicamente a más gasto, sino también a una mejor organización y funcionamiento. Hay universidades que obtienen una calidad superior al gasto que realizan: es el caso de las dos autónomas, de la Complutense y la Universidad de Barcelona. Son universidades grandes, algo masificadas, pero que concentran los mejores recursos organizacionales del país. También destacan por una calidad mayor de lo esperable (según el gasto) las universidades de Santiago, Valencia, Salamanca, Sevilla y Granada. Son todas ellas universidades muy antiguas, que han acumulado recursos pero que actualmente invierten menos de lo previsto en sus estudiantes y que seguramente están masificadas. En el otro lado (a la derecha) están las universidades que gastan mucho pero no obtienen un nivel de calidad tan elevado: son las tres politécnicas y Pública de Navarra. El rendimiento es menor de lo esperado; los estudiantes que terminan la carrera están bien preparados, pero hay un nivel de mortalidad escolar elevado. Se observa un núcleo de universidades de gasto bajo y de calidad proporcionalmente más baja aún: son Burgos, La Rioja, Huelva, Jaén, Almería, Pablo de Olavide y Vigo. Si se observa la distribución de las universidades públicas en el gráfico, se llega a la conclusión de que, más que una recta (de regresión), es una curva. Cuando las universidades gastan poco, su calidad es baja. Incluso cuando empieza a aumentar el gasto, la 


\section{GRÁFICO 2}

Gasto y calidad de las universidades públicas

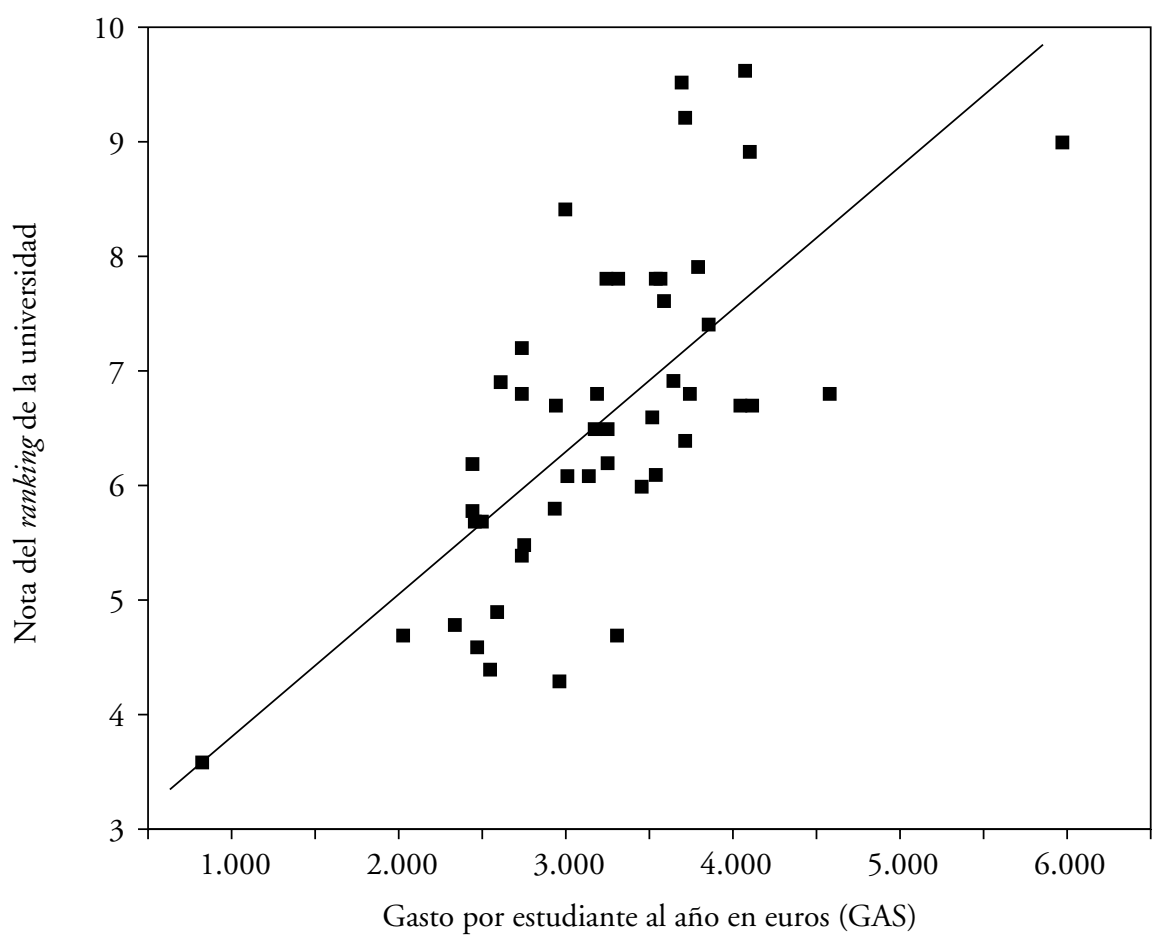

NotA: La correlación entre GAS y nota del ranking es 0,68.

calidad apenas varía ${ }^{7}$. Hacia los 3.000 euros por estudiante al año, la calidad se eleva en algunos casos. A partir de de los 4.500 euros parece que hay un techo de cristal que es difícilmente traspasable. La calidad apenas aumenta aunque se duplique el presupuesto de la universidad. Las dos autónomas y las dos centrales (Complutense y Barcelona) tienen una calidad igual o mejor que la Pompeu Fabra, pero con mucho menos gasto. De aquí es importante concluir que el gasto explica la calidad pero solamente hasta un cierto nivel. La inversión es importante que supere los 4.000 euros por estudiante al año, pero a partir de entonces la calidad depende de otros factores, entre los que están la dedicación a la investigación y al avance del conocimiento y quizás la dotación de perso-

7 Sin embargo, entre las diez universidades públicas españolas, La Rioja es la universidad más cara (705 euros por punto en el ranking) y Burgos la segunda más cara (690 euros por punto). En este sentido la falta de calidad cuesta más. 
nal de administración y servicios (PAS), y la dotación de bibliotecas y ordenadores.

Para cuantificar más exactamente estas relaciones, la tabla 2 presenta las correlaciones de los indicadores básicos de calidad para las universidades públicas. Incluye entre los recursos la variable de gasto por estudiante al año en euros (GAS), que proviene de los datos de la CRUE, y los datos de calidad de las universidades en forma de número de orden (lógicamente, con correlaciones negativas), así como la nota efectiva de cada universidad pública. El gasto depende bastante del nivel de riqueza (PIB) de la región (correlación de 0,56), lo que es esperable. Pero no tiene ninguna relación con la antigüedad (ANT) de las universidades $(0,04)$. Es decir, que las universidades medievales no salen más baratas — ni más caras - que las nuevas en cuanto a gastos corrientes. Eso explica el nivel alto de isomorfismo de las universidades públicas españolas. El gasto se relaciona mucho con los indicadores de recursos pues, lógicamente, dependen del dinero que se emplea en ellos. La correlación es alta con profesorado (GAS con PRO es 0,80 ), con libros de biblioteca $\operatorname{LBE}(0,65)$ y con otro personal de administración y servicios PAS $(0,64)$. Además, el gasto correlaciona, aunque un poco menos, con indicadores de resultados: 0,54 con productividad de las carreras ETC y 0,40 con productividad del doctorado TDE. Lógicamente, el efecto benéfico de los presupuestos es especialmente importante con los recursos, pero se difumina un poco con los resultados obtenidos, pues el output depende también de otras variables difíciles de medir como funcionamiento y organización. Lo importante para la calidad es el resultado final, el output de la organización. Es sorprendente que no haya relación entre el gasto y la proporción de estudiantes en carreras largas (GAS con LES es solamente $0,06)^{8}$. La correlación con mujeres-profesoras MPR es incluso negativa $(-0,21)$. Lo más significativo de esta tabla es que la correlación del gasto con los indicadores de calidad es considerable: 0,68 con la nota del ranking de las universidades públicas, e incluso -0,65 con el número de orden en el ranking ${ }^{9}$.

Las correlaciones con la nota del ranking son casi siempre un poco mayores que con el número de orden, pues lógicamente la nota exacta afina más las diferencias que el orden. Lo significativo del presente estudio es que la correlación entre nota del ranking y número de orden es -0,97. Lógicamente, debe ser alta, pero el que sea tan elevada significa que las universidades públicas son bastante homogé-neas, y que existe un gradiente de universidades (presenciales y públicas) que van desde 9,6 puntos de la Universidad Complutense hasta 4,3 puntos de la Universidad de Burgos, sin que existan saltos grandes. Eso es una indicación adicional de que el presente análisis diferencia la calidad de las universidades, sin separar las universidades en grupos diversos. La idea de que existe un gradiente continuo, y que la calidad correlaciona de forma alta con el

8 Eso es extraño pues supone que el coste de las carreras largas (con segundo ciclo) es casi igual al coste de carreras cortas, con únicamente el primer ciclo. Eso no debería ser así.

9 Es negativa pues cuanto mejor es una universidad, menor es su número de orden. 
TABLA 2

Correlaciones de indicadores de calidad de las universidades públicas

\begin{tabular}{|c|c|c|c|c|c|c|c|c|c|c|c|c|}
\hline \multirow[b]{2}{*}{ Correlaciones } & \multicolumn{2}{|c|}{ Ranking } & \multicolumn{3}{|c|}{ Contexto } & \multicolumn{4}{|c|}{ Recursos } & \multicolumn{2}{|c|}{ Funcionamiento } & \multirow{2}{*}{$\begin{array}{c}\text { Resul- } \\
\text { tados }\end{array}$} \\
\hline & $\begin{array}{l}N .^{o} \text { de } \\
\text { orden }\end{array}$ & Nota & $P I B$ & $A N T$ & FET & $G A S$ & PRO & PAS & $L B E$ & LES & $M P R$ & \\
\hline Nota & $-0,97$ & & & & & & & & & & & \\
\hline PIB & $-0,31$ & 0,37 & & & & & & & & & & \\
\hline ANT & $-0,49$ & 0,44 & $-0,11$ & & & & & & & & & \\
\hline 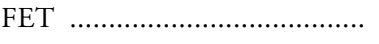 & $-0,48$ & 0,48 & $-0,10$ & 0,64 & & & & & & & & \\
\hline 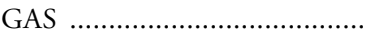 & $-0,65$ & 0,68 & 0,56 & 0,04 & 0,04 & & & & & & & \\
\hline PRO & $-0,59$ & 0,61 & 0,50 & $-0,08$ & $-0,17$ & 0,80 & & & & & & \\
\hline PAS & $-0,55$ & 0,56 & 0,23 & $-0,02$ & $-0,15$ & 0,64 & 0,80 & & & & & \\
\hline LBE & $-0,59$ & 0,63 & 0,34 & 0,27 & 0,14 & 0,65 & 0,56 & 0,62 & & & & \\
\hline 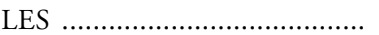 & $-0,54$ & 0,53 & 0,17 & 0,38 & 0,54 & 0,06 & $-0,13$ & $-0,13$ & 0,09 & & & \\
\hline 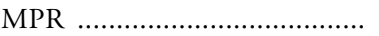 & $-0,07$ & 0,09 & 0,17 & 0,23 & 0,03 & $-0,21$ & $-0,04$ & 0,00 & 0,29 & 0,10 & & \\
\hline ETC & $-0,61$ & 0,65 & 0,11 & 0,31 & 0,36 & 0,54 & 0,43 & 0,45 & 0,37 & 0,09 & $-0,11$ & \\
\hline TDE & $-0,80$ & 0,82 & 0,27 & 0,31 & 0,30 & 0,40 & 0,39 & 0,29 & 0,17 & 0,62 & $-0,03$ & 0,38 \\
\hline
\end{tabular}

NOTA: PIB: producto interior bruto, ANT: antigüedad, FET: número de facultades y escuelas técnias superiores, GAS: gasto por estudiante al año, PRO: profesores por cien estudiantes, PAS: personal de administración y servicios por cien estudiantes, LBE libros de biblioteca por estudiante, LES: proporción de estudiantes en carreras largas, MPR: proporción de mujeres profesoras, ETC: proporción de estudiantes que terminan la carrera en los años justos, TDE: tesis doctorales por cada mil estudiantes al año. 
gasto por estudiante, demuestra que el análisis de calidad funciona bien con las universidades públicas españolas. El caso de las universidades privadas es diferente, y requiere otro artículo ${ }^{10}$. La hipótesis de relación entre gasto y calidad se confirma, pues, con los datos actuales existentes en España. La calidad depende del gasto, aunque el efecto benéfico de más recursos económicos disminuye según aumenta la calidad. Todo sugiere que la calidad adicional resulta más barata que la inicial. Las universidades públicas españolas son bastante diferentes en cuanto a calidad, pero también en relación con su coste ${ }^{11}$.

\begin{abstract}
Up until now in Spain, it was not possible to relate the quality of universities to the cost they involve. At last, in 2002, it is possible to calculate these two variables thanks to the publication of data collected by the National Statistics Institute and the CRUE (Spanish Universities Vice-Chancellor's Conference). The close relationship between quality and cost shown by the data included here makes it necessary to re-examine the relationship between resources and results, and even presumed university autonomy. Bearing in mind that they are different variables, and are calculated independently, the relationship is even more striking. However, there are universities that — relatively speaking - escape from such a causal relationship, which makes it possible to assess the importance of variables of context and functioning. Missing from the Sociology of Organizations are serious studies of the university in Spain. With the new Universities Act (6/2001) and the Entitlement/Access decree (744/2002), the Spanish university system ( $92 \%$ public and $8 \%$ private) is undergoing a greater transformation than sociologists imagine. The information in this research paper summarizes around one hundred variables already analysed in the study entitled Excelencia: Calidad de las universidades españolas (Excellence: The quality of Spanish universities) (published by the CIS in 2001) and thousands of specific facts about public and private universities. Spanish universities are, for the first time, assessed in terms of their productivity and quality, and a diversity which means increasing competition for the best students, teachers/professors and university personnel is observed. This data also makes it possible to progress in an important discussion of the possibility of creating in Spain a model of university-research centre that is competitive at an international level. Unfortunately, such a change is occurring in the middle of a process of university decentralization, and so the design of these (few) international universities-research centres devolves upon the seventeen autonomous regional communities.
\end{abstract}

${ }^{10}$ Es importante el libro de Richard S. Ruch, Higher Ed, Inc. The Rise of the For-Profit University (Baltimore: The Johns Hopkins University Press, 2001), 182 pp. Ver también James J. Duderstadt, A University For the 21st Century (Ann Arbor: The University of Michigan Press, 2000), 358 pp.

${ }^{11}$ Para un análisis comparativo entre países (incluyendo España) véase: Jesús M. de Miguel y Bernabé Sarabia Heydrich, "La Universidad española en un mundo globalizado: Los recursos», REIS (2003). 


\section{TEXTO CLÁSICO}

